\title{
Existence of continuous or constant Finsler's variables for parameter dependent systems
}

\author{
J. Y. Ishihara, H. T. M. Kussaba, R. A. Borges
}

\begin{abstract}
Finsler's lemma is a classic mathematical result with applications in control and optimization. When the lemma is applied to parameter dependent LMIs, as such those that arise from problems of robust stability, the extra variables introduced by this lemma also become dependent on this parameter. This technical note presents some sufficient conditions which ensure, without losing generality, that these extra variables can assume a simple functional dependence on the parameters as continuity or even independence. The results allow avoiding an unnecessary use of a more functionally complicated parameter dependent variable that increases the search computational burden without reducing the conservatism of the solution.
\end{abstract}

Index Terms-Finsler's lemma, Linear matrix inequality.

\section{INTRODUCTION}

Recently, the classical Finsler's lemma was reinterpreted to give novel LMI characterizations to stability and control problems [1]. One important fact that motivates the search for LMI formulations is that nowadays the LMI theory is equipped with a large number of techniques which allows one to extend LMI analysis characterization to control or filtering design conditions in a quite systematic way (see, e.g. [2]). The LMI theory has been successfully applied to state a large number of problems arising from system and control theory as standard convex optimization problems [3]-[5]. Once a problem is stated as an LMI, it can be handled numerically in polynomial time by several software such as the LMI Control Toolbox [6] and SeDuMi [7], and can even, in some cases, be solved analytically [3].

Another advantage of the application of the Finsler's lemma is that it enables one to deal with robustness issues using parameter dependent (PD) formalism [1]. It was observed that certain PD quadratic or nonlinear matricial inequalities can be recast as PD-LMIs through the Finsler's lemma. With this, several robust control problems turned out to be analyzable in PD-LMI context without loss of generality. Prior to the application of Finsler's lemma, in the literature there were nonconservative but very computationally expensive nonlinear inequality solutions or less expensive but approximated and conservative LMI solutions.

However, one should remember that although a PD-LMI formulation is less computationally demanding than a nonlinear inequality formulation, a PD-LMI is still very computationally demanding. It is known that a generic PD-LMI is an NPhard problem [8]. In face of this, there have been several

The authors are with the Department of Electrical Engineering, University of Brasília, Brazil. A portion of this work was completed while J. Y. Ishihara was a visiting associate researcher at Univ. California Los Angeles (UCLA). ishihara@ene.unb.br, kussaba@lara.unb.br, raborges@ene.unb.br. approaches in the linear matrix inequality literature to relax PD-LMIs problems. For instance, one can consider the worst case scenario for the effect of the parameter over a property of interest, such as stability or $\mathscr{H}_{\infty}$ gain. Although this approach is effective, it leads to very conservative criteria. A less conservative but naive method to solve a PD-LMI would be to approximate the parameter space $S$ by a finite set of points-this would relax the PD-LMI into a finite number of LMIs. However, this method usually requires a great number of points for its results to become satisfactory, which generates a heavy computational load. Moreover, if some points are excluded, it is possible that the result turns out to be excessively optimist [9]. Another relaxation procedure is to use a polynomial relaxation, which consists of restricting the variables of the PD-LMI to polynomial functions of a fixed degree $g$ and using the matrix coefficients of the polynomial stemmed from this procedure to generate new LMIs independent of the parameter. With the increase of the degree $g$ of the polynomial, less conservative sets of conditions are obtained. From degrees greater than some value, the set of conditions imply the original PD-LMI [10].

Along with the technique of polynomial relaxation, in some PD-LMI solvable problems, one may also use the Finsler's lemma to relax conservativeness in further analysis. For instance, in the context of robust filter or control design, consider an uncertain continuous-time linear system given by

$$
\dot{x}(t)=A(s) x(t)
$$

where $s$ is a vector of unknown parameters belonging to a known set $S$. It is possible to prove (see [1]) that a sufficient condition to robust stability is related to the existence of a positive definite matrix valued function $P(s)$ and a scalar function $\mu(s)$ such that for all $s \in S$,

$$
\left[\begin{array}{cc}
-\mu(s) A^{T}(s) A(s) & \mu(s) A^{T}(s)+P(s) \\
\mu(s) A(s)+P(s) & -\mu(s) I
\end{array}\right] \prec 0 .
$$

Differently from the classical approach, in (2), the matrix variable $P(s)$ does not multiply the system matrix $A(s)$. This separation allows one to deal with stability analysis of closed loop systems to design stabilizing controllers or filters in a much simpler procedure than the traditional. Also, it may lead to less conservative results [11], [12]. As a consequence, Finsler's lemma has been notably employed in the literature in several contexts [13]-[19]. For examples of several control problems that can be dealt with the Finsler's lemma (also known as S-lemma) one can see [12]. The drawback of this approach is that it increases the search space. For instance, in contrast to the traditional stability analysis with a unique 
variable $P(s)$, (2) is a PD-LMI in $P(s)$ and $\mu(s)$, that is, now the introduced extra function $\mu(s)$ is also a variable that needs to be found.

Relaxation is a good resort when the solution space is little known. Another approach considered in the literature is to find some properties on the parameter set $S$ and on the matrices of the system in order to reduce the search space of the solutions [10], [20]. In fact, if $S$ is a compact set and the matrices of the PD-LMI depend continuously on the parameter, in [10] it is proved that if the PD-LMI has a solution, then one can restrict the search for a solution in the set of polynomial functions. In the particular case that $S$ is a unit simplex, there will also be without loss of generality, a solution that is a homogeneous polynomial [21]. These results are important since, with the knowledge that a continuous function can be approximated by a polynomial, they form the theoretical justification for the polynomial relaxation approach (where at least a continuous solution is supposed to exist). If the parameter space is instead all the space and the matrices of the PD-LMI depends polynomially on the parameter, then [20] shows that one can restrict the search space and look for a rational solution.

Besides the polynomial or rational structures for the slack variable introduced by the Finsler's lemma, we show in this paper that there are some cases in which a parameter independent slack variable is as good as a parameter dependent one. In other words, there is no gain to impose a complicated functional structure to the extra variable. In fact, the objective of this paper is to investigate some conditions in which it is possible, without loss of generality, to impose solutions of simpler functional structures, such as continuous, rational or constant solutions for the slack variables introduced by Finsler's lemma. In the LMI context, this allows reducing computational burden without increasing the conservatism of the solution. This work is an extension of our previous analysis on uniform versions of Finsler's lemma [22]. Here, some new results are presented and some results from [22] were improved: the converse of Lemma 3 from [22] has been proved in Lemma 4 of this paper. Both Lemma 3 and Theorem 2 from [22] were stated for matrix-valued functions of a compact subset of $\mathbb{R}$. In this paper, these results are extended for matrixvalued functions of a compact subset of $\mathbb{R}^{d}$. The hypothesis of Theorem 1 and Corollary 1 from [22] has been weakened in Theorem 4 of this work.

Notation: In the sequel the following notation will be used: $\mathbb{R}$ is the set of real numbers, $\mathbb{R}^{m \times n}$ the set of real matrices of order $m \times n$. $\mathbb{S}^{n}, \mathbb{S}_{>0}^{n}$ and $\mathbb{S}_{>0}^{n}$ are, respectively, the set of symmetric, positive definite and positive-semidefinite matrices of order $n \times n$. For a matrix $A, A \prec 0$ indicates that $-A \in \mathbb{S}_{>0}^{n}$, $A^{T}$ its transpose; $\operatorname{Im}(A)$ and $\operatorname{Ker}(A)$ are respectively the image and the kernel of $A ; A^{\perp}$ is a matrix whose columns span a basis for $\operatorname{Ker}(A)$ and $A^{1 / 2}$ denotes the principal square root of a positive-semidefinite matrix $A$.

\section{THE FINSLER'S LEMMA}

The importance of the Finsler's lemma can be highlighted by the fact that it is equivalent to other important results in control and optimization literature such as Yakubovich's S-lemma [23]. In fact, Finsler's lemma have been proved several times [1], [24]-[29]. Nowadays the Finsler's lemma is commonly stated as below:

Lemma 1. [1] Let $Q \in \mathbb{S}^{n}$ and $B \in \mathbb{R}^{m \times n}$, with $\operatorname{rank}(B)<n$. Then the following statements are equivalent:

1) $x^{T} Q x<0$ for all $x \in \mathbb{R}^{n}$ such that $x \neq 0$ and $B x=0$.

2) There exists $\mu \in \mathbb{R}$ such that $Q-\mu B^{T} B \prec 0$.

3) There exists $X \in \mathbb{R}^{n \times m}$ such that $Q+X B+B^{T} X^{T} \prec 0$.

4) $\left(B^{\perp}\right)^{T} Q B^{\perp} \prec 0$.

The equivalence between (1) and 2) is attributed to Paul Finsler [30], where he considered a more general case involving an indefinite matrix instead of the positive semi-definite matrix $B^{T} B$. It is also interesting to remark that the equivalence between (3) and 4 can be seen as a particular case of the Projection Lemma (also known as Elimination Lemma), which is also widely used in control [3], [31].

The first contribution of this paper is the observation that in statements 2) and 3) of Lemma 1, the hypothesis $\operatorname{rank}(B)<n$ is not necessary.

Lemma 2. Let $Q \in \mathbb{S}^{n}$ and $B \in \mathbb{R}^{m \times n}$, with $\operatorname{rank}(B)=n$. Then there exists $\mu \in \mathbb{R}$ such that $Q-\mu B^{T} B \prec 0$. One such $\mu$ is given by

$$
\mu=1+\frac{\lambda_{\max }(Q)+\left|\lambda_{\max }(Q)\right|}{\lambda_{\min }\left(B^{T} B\right)} .
$$

Moreover, there exists $X \in \mathbb{R}^{n \times m}$ such that $Q+X B+$ $B^{T} X^{T} \prec 0$. One such $X$ is given by

$$
X=-\frac{1}{2}\left[1+\frac{\lambda_{\max }(Q)+\left|\lambda_{\max }(Q)\right|}{\lambda_{\min }\left(B^{T} B\right)}\right] B^{T} .
$$

Proof: See Lemma 7 in Appendix and consider $M=Q$ and $N=B^{T} B$.

With Lemma 2, one can generalize the Finsler's lemma by eliminating the hypothesis $\operatorname{rank}(B)<n$. When $\operatorname{rank}(B)=n$, the empty sentences 10 and (4) are reinterpreted as being trivially satisfied. This general Finsler's Lemma, extended to parameter dependent systems, is stated in the next section.

\section{FINSLER'S LEMMAS FOR PARAMETER DEPENDENT SYSTEMS}

When dealing with uncertain systems, the matrices $Q$ or $B$ can become dependent on parameters [11]. A pointwise extension of Finsler's lemma is easily obtained as stated in the following.

Lemma 3. Let $S \subseteq \mathbb{R}^{d}, Q: S \rightarrow \mathbb{S}^{n}$ and $B: S \rightarrow \mathbb{R}^{m \times n}$. Then the following statements are equivalent:

(F1) For each $s \in S$, one has $x^{T} Q(s) x<0$ for all $x \in \mathbb{R}^{n}$ such that $x \neq 0$ and $B(s) x=0$.

(F2) $(\forall s \in S)(\exists \mu(s) \in \mathbb{R}): Q(s)-\mu(s) B^{T}(s) B(s) \prec 0$.

(F3) For each $s \in S$, there exists $X(s) \in \mathbb{R}^{n \times m}$ such that

$$
Q(s)+X(s) B(s)+B^{T}(s) X^{T}(s) \prec 0 .
$$

(F4) For each $s \in S$, one has that $\left(B^{\perp}(s)\right)^{T} Q(s) B^{\perp}(s) \prec 0$.

Proof: Follows directly extending pointwisely Lemma 1 and Lemma 2 
The inequalities in sentences (F2) and (F3) are known as parameter dependent LMIs (PD-LMIs), and must be satisfied for all parameters $s \in S$. Since the problem of finding a solution to a PD-LMI may be NP-hard [10], it is interesting to reduce the search space by seeking solutions in classes of functions $\mu(s)$ or $X(s)$ with some functional structure like continuity, rational or polynomial dependency or even independent constant solution. Since we can obtain the solution $X(s)=-\frac{1}{2} \mu(s) B^{T}(s)$ for (F3) once we have a solution $\mu(s)$ for (F2), in this paper we will be mainly interested in the following situations for (F2):

(F2a) There exists a function $\mu: S \rightarrow \mathbb{R}$ such that $Q(s)-$ $\mu(s) B^{T}(s) B(s) \prec 0$ for all $s \in S$.

(F2b) There exists a continuous function $\mu: S \rightarrow \mathbb{R}$ such that $Q(s)-\mu(s) B^{T}(s) B(s) \prec 0$ for all $s \in S$.

(F2c) There exists a rational function $\mu: S \rightarrow \mathbb{R}$ without singularities on $S$ such that $Q(s)-\mu(s) B^{T}(s) B(s) \prec 0$ for all $s \in S$.

(F2d) There exists a polynomial function $\mu: S \rightarrow \mathbb{R}$ such that $Q(s)-\mu(s) B^{T}(s) B(s) \prec 0$ for all $s \in S$.

(F2e) There exists a constant $\bar{\mu} \in \mathbb{R}$ such that $Q(s)-$ $\bar{\mu} B^{T}(s) B(s) \prec 0$ for all $s \in S$.

It is obvious that $(\mathrm{F} 2 \mathrm{e}) \Rightarrow(\mathrm{F} 2 \mathrm{~d}) \Rightarrow(\mathrm{F} 2 \mathrm{c}) \Rightarrow(\mathrm{F} 2 \mathrm{~b}) \Rightarrow(\mathrm{F} 2 \mathrm{a})$, however, as shown in the following counterexample, reverse implications are not true in general and additional hypothesis on the set $S$ and the functions $Q(s)$ and $B(s)$ are necessary to assure that existence of pointwise solutions $\mu(s)$ guarantees the existence of a solution with a simple functional dependence on $s$.

Example 1. For $S=(0,+\infty) \subset \mathbb{R}$, let $Q: S \rightarrow \mathbb{R}^{2 \times 2}$ and $B$ : $S \rightarrow \mathbb{R}^{1 \times 2}$ be given by

$$
Q(s)=\left[\begin{array}{cc}
-1 & 0 \\
0 & q(s)
\end{array}\right], B^{T}(s)=\left[\begin{array}{l}
0 \\
s
\end{array}\right] .
$$

One has that any solution $\mu(s)$ for $Q(s)-\mu(s) B^{T}(s) B(s) \prec 0$ must satisfy

$$
\mu(s)>\frac{q(s)}{s^{2}}, s \in(0,+\infty) .
$$

Thus, independently if the function $q(s)$ is discontinuous or not, to guarantee that (F2a) will be satisfied one can take $\mu(s)=\frac{q(s)}{s^{2}}+\varepsilon$ with $\varepsilon>0$.

For $q(s)=s$, one function satisfying (F2b) and (F2c) is $\mu(s)=\frac{1}{s}+\varepsilon$ with $\varepsilon>0$. It is easy to see that there is no constant solution $\mu(s)=\bar{\mu}$ nor a polynomial solution $\mu(s)=$ $a_{n} s^{n}+\ldots+a_{1} s+a_{0}$ since in (3) the function $\frac{q(s)}{s^{2}}=\frac{1}{s}$ grows without bound as $s$ goes to 0 .

Similarly, for $q(s)=e^{s}$, one function satisfying (F2b) is $\mu(s)=\frac{e^{s}}{s^{2}}+\varepsilon$ with $\varepsilon>0$. And it can be shown that there is no constant, polynomial nor rational solution $\mu(s)$, since otherwise from (3) there would exist a rational function growing faster than the exponential as $s \rightarrow \infty$.

Further, take a function $q(s)$ such that, for some point $0<$ $\bar{s}<+\infty, \frac{q(s)}{s^{2}}$ goes to $+\infty$ for $s \downarrow \bar{s}$ and goes to any value $\bar{q}<+\infty$ for $s \uparrow \bar{s}$. In this case, it is impossible to find a continuous $\mu(s)$ satisfying the inequality (3), that is, although (F2a) is satisfied, (F2b) is not.
The main objective of this paper is to investigate under which conditions there are equivalences among the statements (F2a),...,(F2e) since this means that one can reduce the search space to subspaces of functions $\mu(s)$ with a simpler structure without loss of generality. Some of these equivalences have already been shown in the literature.

Theorem 1. [20] If the functions $Q(s)$ and $B(s)$ are polynomial (matrices) over $S=\mathbb{R}^{d}$, then $(F 2 a)$ is equivalent to $(F 2 d)$.

Proof: Follows directly by choosing $F=-Q$ and $G=$ $-B^{T} B$ in Proposition 3.2 from [20].

Theorem 2. [10] Let $Q: S \rightarrow \mathbb{S}^{n}$ and $B: S \rightarrow \mathbb{R}^{m \times n}$ be continuous matrix valued functions on a compact $S \subset \mathbb{R}^{d}$. Then $(F 2 a)$ is equivalent to $(F 2 d)$.

Proof: Follows directly from Theorem 1 of [10].

In the next section, we investigate other conditions for some equivalences among the statements (F2a),..,(F2e). In particular, one of the main goals of this paper is to investigate when the PD-LMI in (F2) is also valid uniformly in $\mu$, that is, when $(\mathrm{F} 2 \mathrm{a})$ is equivalent to $(\mathrm{F} 2 \mathrm{e})$.

\section{A. Results on the scalar-valued function $\mu(s)$}

The first result of this paper shows that if the parameter set $S$ is compact, then there is no gain in searching for a complicated continuous solution $\mu(s)$ since one can reduce the search to constant solutions.

Theorem 3. Let $Q: S \rightarrow \mathbb{S}^{n}$ and $B: S \rightarrow \mathbb{R}^{m \times n}$ be matrix valued functions on a compact $S \subset \mathbb{R}^{d}$. Then the sentences from $(F 2 b)$ to $(F 2 e)$ are all equivalent. If further, $Q$ and $B$ are continuous then the sentences from $(F 2 a)$ to $(F 2 e)$ are all equivalent.

Proof: The proof for (F2e) $\Rightarrow(\mathrm{F} 2 \mathrm{~d}) \Rightarrow(\mathrm{F} 2 \mathrm{c}) \Rightarrow(\mathrm{F} 2 \mathrm{~b})$ is immediate. Suppose now that (F2b) is valid. By Weierstrass' theorem [32, p.90], the function $\mu(s)$ has a maximum $\mu_{\max }$ in $S$. Thus

$$
0 \succ Q(s)-\mu(s) B^{T}(s) B(s) \succeq Q(s)-\mu_{\max } B^{T}(s) B(s)
$$

and (F2e) is satisfied with $\bar{\mu}=\mu_{\max }$. If $Q$ and $B$ are continuous, then (F2a) is equivalent to (F2d) by Theorem 2. Since all sentences from $(\mathrm{F} 2 \mathrm{~b})$ to $(\mathrm{F} 2 \mathrm{e})$ are equivalent, the result follows.

Note that for PD-LMIs with one variable $\mu(s)$, Theorem 3 extends the consecrated result of [10]. This might be useful in the context of polynomial relaxation procedures for PD-LMIs [10], [21], wherein it is assumed that $\mu(s)$ is a polynomial function. With Theorem 3, one can reduce the search space from the set of polynomial functions to the set of real numbers.

Another fundamental lemma which gives a general condition to guarantee the existence of one $\mu$ for all $s \in S$ is presented below.

\footnotetext{
${ }^{1}$ The original Proposition 3.2 establishes the existence of $\mu$ rational without singularities. However, from its proof, one can conclude that $\mu$ can be chosen polynomial. We thank the anonymous reviewer that pointed out the possibility of this extension and to prof. Jaka Cimprič for ratifying this fact.
} 
Lemma 4. Let $Q: S \rightarrow \mathbb{S}^{n}$ and $B: S \rightarrow \mathbb{R}^{m \times n}$ be functions on $S \subset \mathbb{R}^{d}$ such that

$$
\sup _{s \in S} \inf \left\{\mu \in \mathbb{R} \mid Q(s)-\mu B^{T}(s) B(s) \prec 0\right\}<\infty .
$$

Then the statements from $(F 2 a)$ to $(F 2 e)$ are all equivalent. Conversely, if (F2e) holds, then (F2a) and (4) hold.

Proof: The proof for (F2e) $\Rightarrow(\mathrm{F} 2 \mathrm{~d}) \Rightarrow(\mathrm{F} 2 \mathrm{c}) \Rightarrow(\mathrm{F} 2 \mathrm{~b}) \Rightarrow$ (F2a) is immediate. We now prove that (F2a) implies (F2e). For each $s \in S$, define

$$
\mathscr{M}(s)=\left\{\mu \in \mathbb{R} \mid Q(s)-\mu B^{T}(s) B(s) \prec 0\right\} .
$$

For each $s$, one has that if $\mu^{*} \in \mathscr{M}(s)$, then $\mu^{*}+\alpha \in \mathscr{M}(s)$ for all $\alpha \geq 0$, since

$$
0 \succ Q(s)-\mu^{*} B^{T}(s) B(s) \succeq Q(s)-\left(\mu^{*}+\alpha\right) B^{T}(s) B(s) .
$$

By (F2a), one has that $\mathscr{M}(s) \neq \emptyset$ for any $s \in S$. Therefore, there always exists a $\mu^{*}(s) \in \mathscr{M}(s)$ such that $\left[\mu^{*}(s),+\infty\right) \subseteq$ $\mathscr{M}(s)$. By (4), there exists $m \in \mathbb{R}$ such that

$$
\inf \left\{\mu \in \mathbb{R} \mid Q(s)-\mu B^{T}(s) B(s) \prec 0\right\}<m, \forall s \in S .
$$

Consequently, $m \in \mathscr{M}(s)$ for all $s \in S$ and $\bar{\mu}=m$ is such that (F2e) holds. Conversely, suppose that (F2e) holds. It is clear that (F2a) holds and $\mathscr{M}(s) \neq \emptyset$ for all $s \in S$ since $\bar{\mu} \in \mathscr{M}(s)$. It follows that, $\inf \mathscr{M}(s) \leq \bar{\mu}, \forall s \in S$ and $\sup _{s \in S} \inf \mathscr{M}(s) \leq$ $\bar{\mu}<\infty$.

Remark 1. If one adopts the convention that $\inf \emptyset=+\infty$ then Lemma 4 can be restated as: (F2e) is equivalent to (4). The above presentation of the lemma was preferred in order to distinguish the situations where does not exist $\mu(s)$ from those where the function $\mu(s)$ goes to infinity.

Note that Lemma 4 is very general and does not require any special structure on the functions $Q(s)$ and $B(s)$ nor on the set $S$. One immediate use of Lemma 4 is a derivation of a Finsler's lemma version for switching systems. This is a case where (4) can be easily checked as discussed in the following.

Corollary 1. Consider matrix valued functions $Q: S \rightarrow \mathbb{S}^{n}$ and $B: S \rightarrow \mathbb{R}^{m \times n}$ assuming a finite number of values given by the set $\left\{\left(Q_{1}, B_{1}\right), \ldots,\left(Q_{N}, B_{N}\right)\right\}$. Then the statements from ( $\left.F 2 a\right)$ to $(F 2 e)$ and the following statements are equivalent:

(F2f) There exists a constant $\bar{\mu} \in \mathbb{R}$ such that $Q_{i}-\bar{\mu} B_{i}^{T} B_{i} \prec 0$ for every $i \in\{1, \ldots, N\}$.

(F2g) For every $i \in\{1, \ldots, N\}, \exists \mu_{i} \in \mathbb{R}$ such that $Q_{i}-\mu_{i} B_{i}^{T} B_{i} \prec$ 0 .

Furthermore, if $Q(s)$ and $B(s)$ are piecewise constant functions on $S$, then $(F 2 a)-(F 2 g)$ are also equivalent to

(F2h) There exists a piecewise constant function $\mu: S \rightarrow \mathbb{R}$ such that $Q(s)-\mu(s) B^{T}(s) B(s) \prec 0$ for all $s \in S$.

Proof: Consider (F2a) valid. It is clear that for every $i \in\{1, \ldots, N\}, \quad \mathscr{M}_{i}:=$ $\left\{\mu \in \mathbb{R} \mid Q_{i}-\mu B_{i}^{T} B_{i} \prec 0\right\} \neq \emptyset$ and so $\bar{\mu}_{i}:=\inf \mathscr{M}_{i}<+\infty$. Then, $\quad \sup _{s \in S} \inf \left\{\mu \in \mathbb{R} \mid Q(s)-\mu B^{T}(s) B(s) \prec 0\right\}=$ $\sup \left\{\bar{\mu}_{1}, \ldots, \bar{\mu}_{N}\right\}<+\infty$. From Lemma 4 it follows that $(\mathrm{F} 2 \mathrm{a}) \Longrightarrow(\mathrm{F} 2 \mathrm{e})$. The implications $(\mathrm{F} 2 \mathrm{e}) \Rightarrow(\mathrm{F} 2 \mathrm{f}) \Rightarrow$ $(\mathrm{F} 2 \mathrm{~g}) \Rightarrow(\mathrm{F} 2 \mathrm{a})$ and $(\mathrm{F} 2 \mathrm{~d}) \Rightarrow(\mathrm{F} 2 \mathrm{c}) \Rightarrow(\mathrm{F} 2 \mathrm{~b}) \Rightarrow(\mathrm{F} 2 \mathrm{a})$ are immediate. For the last statement of the corollary, define the set $S_{i}:=\left\{s \in S \mid(Q(s), B(s))=\left(Q_{i}, B_{i}\right)\right\}$, consider any $\mu_{i} \in\left\{\mu \in \mathbb{R} \mid Q_{i}-\mu B_{i}^{T} B_{i} \prec 0\right\}$ and $\mu(s)=\mu_{i}, s \in S_{i}$.

One particular case of interest for the Corollary 1 is when the system parameters $Q(s)$ and $B(s)$ represent a switching system, and the parameter set is interpreted as continuous time, $S=\mathbb{R}$, or as discrete time, $S=\mathbb{Z}$. Note that if $\left(Q_{i}, B_{i}\right)$ are appropriately chosen, then the Corollary 11 states that, no matter which switching policy is used, there always exists a continuous or even a constant solution $\mu$. The derivation of Finsler's lemma versions for switching systems is now immediate. One such lemma is given in the following.

Lemma 5. Consider matrix sequences $Q(k) \in$ $\left\{Q_{1}, \ldots, Q_{N_{Q}}\right\} \subset \mathbb{S}^{n}$ and $B(k) \in\left\{B_{1}, \ldots, B_{N_{B}}\right\} \subset \mathbb{R}^{m \times n}$, $k=1,2, \ldots$. Then the following statements are equivalent:

(i) For each $k=1,2, \ldots$, one has $x^{T} Q(k) x<0$ for all $x \in \mathbb{R}^{n}$ such that $x \neq 0$ and $B(k) x=0$.

(ii) There exists a constant $\bar{\mu} \in \mathbb{R}$ such that $Q_{i}-\bar{\mu} B_{j}^{T} B_{j} \prec 0$ for every $i \in\left\{1, \ldots, N_{Q}\right\}$ and for every $j \in\left\{1, \ldots, N_{B}\right\}$.

The next theorem gives conditions for the existence of a continuous solution when it is known that a pointwise solution exists.

Theorem 4. Let $Q: S \rightarrow \mathbb{S}^{n}$ and $B: S \rightarrow \mathbb{R}^{m \times n}$ be continuous functions on $S \subseteq \mathbb{R}^{d}$. Then the statements $(F 2 a)$ and $(F 2 b)$ are equivalent. If further, $S$ is a compact set, then the statements from $(F 2 a)$ to $(F 2 e)$ are all equivalent.

Proof: Define the function $\mu_{\mathrm{inf}}: S \rightarrow \mathbb{R} \cup$ $\{-\infty\} \quad$ as $\quad \mu_{\text {inf }}(s) \quad:=\inf \mathscr{M}(s) \quad$ where $\quad \mathscr{M}(s):=$ $\left\{\mu \in \mathbb{R} \mid Q(s)-\mu B^{T}(s) B(s) \prec 0\right\}$. By $\quad(\mathrm{F} 2 \mathrm{a})$, that is, non emptiness of $\mathscr{M}(s)$, it is clear that $\mu_{\text {inf }}(s)<+\infty$ for all $s \in S$ and $\mu_{\text {inf }}(s)=-\infty$ for $s \in S$ at which $Q(s) \prec 0$ and $B(s)=0$. Since $\bar{\mu} \in \mathscr{M}(s)$ implies that $\bar{\mu}+\alpha \in \mathscr{M}(s)$ for any $\alpha>0$, the set $\mathscr{M}(s)$ is an open interval of the form $\mathscr{M}(s)=\left(\mu_{\text {inf }}(s),+\infty\right)$. Taking the induced topology of $\mathbb{R}^{d}$ on $S$, one can use the same arguments in the proof for continuity on $\mathbb{R}^{d}$ of Lemma 3.1 of [20] to conclude that the extended real-valued function $\mu_{\text {inf }}$ is continuous on $S$.

Now, take any $\varepsilon>0$ and define the real valued function $\tilde{\mu}(s):=\max \left\{\mu_{\mathrm{inf}}(s)+\varepsilon, 0\right\}$. It is clear that $\tilde{\mu}: S \rightarrow \mathbb{R}$ is a continuous function with $\tilde{\mu}(s) \in \mathscr{M}(s)$ for all $s \in S$. Thus (F2b) is satisfied with $\tilde{\mu}$.

Theorem 4 states that a continuous solution exists if $Q$ and $B$ are continuous over an arbitrary subset $S \subseteq \mathbb{R}^{d}$. In contrast, Theorem 2 allows a polynomial solution but just when $S$ is compact. Theorem 1 allows a polynomial solution if the functions $Q$ and $B$ are polynomials over $S=\mathbb{R}^{d}$.

The next example illustrates an application of Theorem 4 in the context of non-linear systems.

Example 2. Consider

$$
\dot{x}=f(x, t)+B^{T}(x, t) u, t>0
$$

where $x \in \mathbb{R}^{n}$ and $u \in \mathbb{R}^{m}$ are the state and the control variables, respectively. The functions $f: \mathbb{R}^{n} \times \mathbb{R}^{+} \rightarrow \mathbb{R}^{n}$ and $B: \mathbb{R}^{n} \times$ $\mathbb{R}^{+} \rightarrow \mathbb{R}^{m \times n}$ are assumed to be smooth. In [33] it is shown that system (5) is universally exponentially stabilizable with 
rate $\lambda$ if there exists a positive definite matrix valued function $M: \mathbb{R}^{n} \times \mathbb{R}^{+} \rightarrow \mathbb{S}_{>0}^{n}$ and a function $\rho: \mathbb{R}^{n} \times \mathbb{R}^{+} \rightarrow \mathbb{R}$ such that

$$
\dot{M}+\left(\frac{\partial f}{\partial x}\right)^{T} M+M\left(\frac{\partial f}{\partial x}\right)-\rho M B^{T} B M+2 \lambda M \preceq 0
$$

for all $x \in \mathbb{R}^{n}$ and $t>0$.

Since the direct solution to (6) is very hard to be obtained, for simplicity, one may consider a restricted search space by choosing $M=I$. This results in

$$
Q(x)-\rho(x) B^{T}(x) B(x) \preceq-2 \lambda I \prec 0
$$

with

$$
Q(x):=\left(\frac{\partial f}{\partial x}\right)^{T}+\left(\frac{\partial f}{\partial x}\right)
$$

Note that even in this simplified context, the search space is still very big since, in principle, $\rho(x)$ could have any structure. Theorem 4 can be called to reduce the search space since it guarantees that there is no loss of generality to assume that a continuous solution $\rho(x)$ exists. In fact, consider, for example, (5) with

$$
f(x, t)=\left[\begin{array}{c}
-e^{x_{1}}+x_{2}^{3} \\
x_{1}
\end{array}\right], B^{T}(x, t)=\left[\begin{array}{l}
0 \\
1
\end{array}\right], Q(x)=\left[\begin{array}{cc}
-e^{x_{1}} & 1+3 x_{2}^{2} \\
1+3 x_{2}^{2} & 0
\end{array}\right] .
$$

One has that the continuous function $\rho(x)=e^{-x_{1}}$ guarantees that (7) holds for all $x \in \mathbb{R}^{2}$ and then, the system is universally exponentially stabilizable.

One interesting consequence of considering continuity of the Finsler's parameter with non-compact $S$ or non-polynomial $Q(s)$ and $B(s)$ is that it opens the investigation of relaxation in more general contexts. In fact, the property that a continuous function can be uniformly approximated by polynomial functions on compact subsets is what guarantees that one can reduce the search space from the set of continuous functions to the set of polynomial functions. For more general subsets, it is known that without compactness but under some other technical conditions, a continuous function can be uniformly approximated by rational functions [34, pp.293-295] or still by functions with some other specific structure [35].

For general sets $S$ or functions $Q(s)$ and $B(s)$, it is very hard to check (47). Nevertheless bounds on $Q(s)$ and $B(s)$ can be used for simpler tests as presented in the next lemma.

Lemma 6. Let $Q: S \rightarrow \mathbb{S}^{n}$ and $B: S \rightarrow \mathbb{R}^{m \times n}$ be functions on $S \subset \mathbb{R}^{d}$ and let $\ell_{Q}, \ell_{R}, u_{Q}, u_{R}: S \rightarrow \mathbb{R}$ such that for all $s \in S$,

$\ell_{Q}(s) I_{n} \preceq Q(s) \preceq u_{Q}(s) I_{n}, \quad \ell_{R}(s) I_{n} \preceq B^{T}(s) B(s) \preceq u_{R}(s) I_{n}$.

Then, a necessary condition for (4) is

$$
\sup _{s \in S} \inf \left\{\mu \in \mathbb{R}_{\geq 0} \mid \ell_{Q}(s)-\mu u_{R}(s)<0\right\}<\infty
$$

and a sufficient condition for (4) is

$$
\sup _{s \in S} \inf \left\{\mu \in \mathbb{R}_{\geq 0} \mid u_{Q}(s)-\mu \ell_{R}(s)<0\right\}<\infty .
$$

In particular, if $Q$ and $B$ are scalar functions, a necessary and sufficient condition for (4) is

$$
Q(s)<0, \forall s \in S_{0} \quad \text { and } \quad \sup _{s \in S_{+}} \frac{Q(s)}{B^{2}(s)}<\infty
$$

where $S_{0}=\{s \in S \mid B(s)=0\}$ and $S_{+}=S \backslash S_{0}$.

Proof: For $(s, \mu) \in S \times \mathbb{R}$, define $\alpha(s, \mu)=$ $\left[\ell_{Q}(s)-\mu u_{R}(s)\right] I_{n}, \quad \beta(s, \mu)=Q(s)-\mu B^{T}(s) B(s) \quad$ and $\gamma(s, \mu)=\left[u_{Q}(s)-\mu \ell_{R}(s)\right] I_{n}$. For each $s \in S$, we have that $\alpha(s, \mu) \preceq \beta(s, \mu) \preceq \gamma(s, \mu)$, for all $\mu \geq 0$. Therefore

$$
\begin{aligned}
\{\mu \geq 0 \mid \gamma(s, \mu) \prec 0\} & \subseteq\{\mu \geq 0 \mid \beta(s, \mu) \prec 0\} \\
& \subseteq\{\mu \geq 0 \mid \alpha(s, \mu) \prec 0\} .
\end{aligned}
$$

[Sufficiency] From (10),,$+\infty>\sup _{s \in S} \inf \{\mu \geq 0 \mid \gamma(s, \mu) \prec$ $0\} \geq \sup _{s \in S} \inf \{\mu \geq 0 \mid \beta(s, \mu) \prec 0\} \geq \sup _{s \in S} \inf \{\mu \in \mathbb{R} \mid$ $\beta(s, \mu) \prec 0\}$.

[Necessity] Since $\sup _{s \in S} \inf \{\mu \in \mathbb{R} \mid \beta(s, \mu) \prec 0\}<+\infty$, exists $m>0$ such that $\inf \{\mu \in \mathbb{R} \mid \beta(s, \mu) \prec 0\}<m, \forall s$. Therefore, $m \in\{\mu \in \mathbb{R} \mid \beta(s, \mu) \prec 0\}, \forall s$. Since $m>0$, we also have that $m \in\{\mu \geq 0 \mid \beta(s, \mu) \prec 0\}, \forall s$. From (10) it follows that $m \in\{\mu \geq 0 \mid \alpha(s, \mu) \prec 0\}, \forall s$. Therefore, $\quad \inf \{\mu \geq 0 \mid \alpha(s, \mu) \prec 0\} \leq m, \forall s \quad$ and $\sup _{s \in S} \inf \{\mu \geq 0 \mid \alpha(s, \mu) \prec 0\} \leq m<+\infty$.

Different from the previous results, the next theorem presents a simple case where it is possible to assure the existence of a solution.

Theorem 5. Let $Q: S \rightarrow \mathbb{S}^{n}$ and $B: S \rightarrow \mathbb{R}^{m \times n}$ be matrix valued functions on $S \subseteq \mathbb{R}^{d}$. If there are functions $\ell_{R}, u_{Q}: S \rightarrow \mathbb{R}$ such that for all $s \in S$,

$$
0 \prec \ell_{R}(s) I_{n} \preceq B^{T}(s) B(s), \quad Q(s) \preceq u_{Q}(s) I_{n},
$$

then the statement $(F 2 a)$ holds. If further, $\ell_{R}$ and $u_{Q}$ are continuous then the statement ( $F 2 b)$ holds. Moreover, if further $\ell_{R}$ and $u_{Q}$ are continuous and $S$ is compact, then all the statements from ( F2a) to ( F2e) hold. In particular, one solution to $(F 2 e)$ is

$$
\bar{\mu}=\sup _{s \in S} \frac{u_{Q}(s)+\left|u_{Q}(s)\right|}{\ell_{R}(s)}+1 .
$$

Proof: Since $\ell_{R}(s)>0$, it follows that $B(s)$ is full column rank for each $s \in S, B^{T}(s) B(s) \in \mathbb{S}_{>0}^{n}$. Thus, the function $\mu(s)$ obtained by extending Lemma 7 pointwisely is such that $Q(s)-\mu(s) B^{T}(s) B(s) \prec 0$ and therefore (F2a) holds. If the functions $\ell_{R}$ and $u_{Q}$ are continuous, then $\mu(s)$ is continuous on $S$. If in addition $S$ is compact, then by Theorem 3 the statements from (F2a) to (F2e) are all equivalent.

Theorem 5 can be used for determining continuous solution $\mu(s)$ even in the case where the matrix valued functions $Q(s)$ and $B(s)$ are not continuous. It is only necessary to find continuous bounding functions $\ell_{R}(s)$ and $u_{Q}(s)$. In the case where the functions $Q(s)$ and $B(s)$ are continuous, one can choose $\ell_{R}(s)=\lambda_{\min }\left(B^{T}(s) B(s)\right), u_{Q}(s)=\lambda_{\max }(Q(s))$ and then, the following corollary can be stated.

Corollary 2. Let $Q: S \rightarrow \mathbb{S}^{n}$ and $B: S \rightarrow \mathbb{R}^{m \times n}$ be matrix valued functions on $S \subseteq \mathbb{R}^{d}$ with $B(s)$ full column rank for every $s \in S$. Then the statement (F2a) holds. If further, $Q$ and $B$ are continuous then the statement (F2b) holds. Moreover, if further $Q$ and $B$ are continuous and $S$ is compact, then all the statements from (F2a) to (F2e) hold. In particular, one solution to $(F 2 e)$ is

$$
\bar{\mu}=\sup _{s \in S} \frac{\lambda_{\max }(Q(s))+\left|\lambda_{\max }(Q(s))\right|}{\lambda_{\min }\left(B^{T}(s) B(s)\right)}+1 .
$$


Other combinations considering constant, polynomial, etc. functional dependence of bounding functions $\ell_{R}(s)$ and $u_{Q}(s)$ can be trivially obtained. In the next section we comment on how the above theorems can be applied to give simpler functional solutions $X(s)$ for the PD-LMI in (F3).

\section{B. Consequences for the matrix-valued function $X(s)$}

In many control problems, one may be led to a PD-LMI in the (F3) formalism, that is, to the problem of finding a matrix function $X: S \rightarrow \mathbb{R}^{n \times m}$ satisfying (F3).

It is known that the general results of Theorem 11 and Theorem 2 are valid for (F3) in the sense that, under their hypotheses, if there is a solution to (F3) then there also exists a rational or a polynomial solution, respectively.

Remembering that if $\mu(s)$ is a solution to (F2) then $X(s)=$ $-\frac{1}{2} \mu(s) B^{T}(s)$ is a solution to (F3), it is easy to apply the results of Section $\amalg I I-A$ to give some sufficient conditions that allow simple functional dependence like continuity or polynomial dependence on $s$ for the variable $X(s)$ in (F3) without loss of generality. Among these possible extensions, one may point out the next theorem which deals with a case closely related to [10] and [20].

Theorem 6. Let $Q: S \rightarrow \mathbb{S}^{n}$ and $B: S \rightarrow \mathbb{R}^{m \times n}$ be matrix valued functions on $S \subseteq \mathbb{R}^{d}$. Suppose that $B(s)$ is a polynomial matrix.

If $S=\mathbb{R}^{d}$ and $Q$ is polynomial, it follows that if there exists a solution $X: \mathbb{R}^{d} \rightarrow \mathbb{R}^{n \times m}$ to (F3), then there is also a solution $\bar{X}: \mathbb{R}^{d} \rightarrow \mathbb{R}^{n \times m}$ that is a polynomial matrix.

If $S \subset \mathbb{R}^{d}$ is compact, $Q$ is continuous and $B$ is polynomial of degree $g$, it follows that if there exists a solution $X: S \rightarrow \mathbb{R}^{n \times m}$ to (F3), then there is also a solution $\bar{X}: S \rightarrow \mathbb{R}^{n \times m}$ that is a polynomial matrix of degree $g$. In particular, if $g=0$, then $\bar{X}$ can be taken as constant.

Proof: If there exists a matrix valued function $X: S \rightarrow$ $\mathbb{R}^{n \times m}$ such that (F3) holds, then it follows directly from Lemma 3 that there exists a function $\mu: S \rightarrow \mathbb{R}$ satisfying (F2a).

Suppose that $S=\mathbb{R}^{d}$ and $Q$ is polynomial. Since $Q$ and $B$ are polynomial matrices over $\mathbb{R}^{d}$, by Theorem 1 it follows that there exists a polynomial $\bar{\mu}$ satisfying (F2d). By taking $\bar{X}(s)=-\frac{1}{2} \bar{\mu}(s) B^{T}(s)$, we have that $\bar{X}$ is a polynomial matrix.

Suppose now that $S \subset \mathbb{R}^{d}$ is compact. Since $Q$ and $B$ are continuous functions and $S$ is compact, by Theorem 4 it follows that there exists a constant $\bar{\mu} \in \mathbb{R}$ satisfying (F2e). The result follows now by taking $\bar{X}(s)=-\frac{1}{2} \bar{\mu} B^{T}(s)$.

In contrast to [20], this theorem presents a case where beside knowing the existence of a polynomial solution, it is also possible to define its degree. This result is useful to reduce the search space to polynomial solutions of degree less than or equal to some specific degree, as the next example illustrates. The example concerns how Finsler's lemma is applied along relaxation techniques to transform a PD-LMI into a set of LMIs. This technique has been applied, for instance in [12], where the effectiveness of this approach is illustrated in the evaluation of LMI methods for robust performance analysis of closed-loop longitudinal dynamics of a civil aircraft.
Example 3. Consider a linear system with polytopic uncertainties, that is,

$$
\dot{x}=A(\alpha) x(t),
$$

with $A(\alpha)=\sum_{i=1}^{N} \alpha_{i} A_{i}$, where $A_{i} \in \mathbb{R}^{n \times n}$ are the vertices of $A(\alpha)$ and $\alpha \in \Delta_{N}:=\left\{\theta \in \mathbb{R}^{N} \mid \sum_{i=1}^{N} \theta_{i}=1, \theta_{i} \geq 0\right\}$.

The system (11) is robustly stable if and only if there exists $P: \Delta_{N} \rightarrow \mathbb{S}^{n}$ satisfying $P(\alpha) \succ 0$ and

$$
A^{T}(\alpha) P(\alpha)+P(\alpha) A(\alpha) \prec 0
$$

for all $\alpha \in \Delta_{N}$. In order to relax this PD-LMI into a finite set of LMIs one can impose a polynomial structure with degree $g$ in $P(\alpha)$ [11] (note that there is no loss of generality in using a polynomial structure by Theorem 2). For instance, considering that $g=1, P(\alpha)=\sum_{i=1}^{N} \alpha_{i} P_{i}$ yields the PD-LMIs $\sum_{i=1}^{N} \alpha_{i} P_{i} \succ 0$ and

$$
\sum_{i=1}^{N} \alpha_{i}^{2}\left(A_{i}^{T} P_{i}+P_{i} A_{i}\right)+\sum_{i=1}^{N-1} \sum_{j=i+1}^{N} \alpha_{i} \alpha_{j}\left(A_{i}^{T} P_{j}+P_{j} A_{i}\right) \prec 0
$$

and, consequently, a sufficient condition to the robust stability of system (11) is that the following set of LMIs is satisfied:

$$
P_{i} \succ 0, A_{i}^{T} P_{j}+P_{j} A_{i}, i, j=1, \ldots, N .
$$

As shown in [11], in order to obtain a possible less conservative set of LMIs that implies the robust stability of (11), instead of applying the relaxation procedure to the standard Lyapunov PD-LMI (12), the relaxation can be applied by imposing a polynomial structure with degree $g$ in the variables $P(\alpha)$ and $X(\alpha)$ of the PD-LMIs $P(\alpha) \succ 0$ and

$$
\left[\begin{array}{cc}
0 & P(\alpha) \\
P(\alpha) & 0
\end{array}\right]+X(\alpha)[A(\alpha) \quad-I]+\left[\begin{array}{c}
A^{T}(\alpha) \\
-I
\end{array}\right] X^{T}(\alpha) \prec 0 .
$$

In this case, there are

$$
n(5 n+1)(N+g-1) ! /(2 g !(N-1) !)
$$

LMI scalar variables [11]. However, by using Theorem 6 the search of a polynomial solution to $X(\alpha)$ can be reduced, without loss of generality, to the search of linear solutions. This leads to

$$
n(n+1)(N+g-1) ! /(2 g !(N-1) !)+2 n^{2} N
$$

scalar variables, which represents a search space reduction of $n^{2} O\left(N^{g}\right)$ scalar variables.

\section{CONCLUSION}

In this technical note, it was proposed a set of sufficient conditions which allows the use of simple structures in the extra variable introduced by Finsler's lemma in the context of parameter dependent matrices. Since an unnecessarily complicated structure of parameter dependent variables increases the computational burden without reducing the conservatism, the results of this note may contribute to the reduction of the computational costs for stability analysis, controller and filter design, and any other parameter dependent results that can be built upon the Finsler's lemma for parameter dependent systems. While some progress has been made in the extra scalar variable, various important issues ask for further efforts. Among them, conditions for smooth or analytic scalar 
variables, more general and less conservative conditions for continuous or polynomial solutions for the extra matrix variable, and extended versions of the Finsler's lemma such as the Projection Lemma, seem essential for extending optimization, control and filter design techniques for parameter dependent systems.

\section{APPENDIX}

Lemma 7. Let $M \in \mathbb{S}^{n}$ and $N \in \mathbb{S}_{>0}^{n}$. Then there exists $\mu \in \mathbb{R}$ such that $M-\mu N \prec 0$. In fact, one such $\mu$ is given by

$$
\mu=\frac{u_{M}+\left|u_{M}\right|}{\ell_{N}}+1
$$

where $\ell_{N}$ and $u_{M}$ are any real numbers such that $0 \prec \ell_{N} I_{n} \preceq$ $N$ and $M \preceq u_{M} I_{n}$. In particular, for $\ell_{N}=\lambda_{\min }(N)$ and $u_{M}=$ $\lambda_{\max }(M)$ one has

$$
\mu=\frac{\lambda_{\max }(M)+\left|\lambda_{\max }(M)\right|}{\lambda_{\min }(N)}+1 .
$$

Proof: Since $N \succ 0$, one has that there exists $\ell_{N} \in \mathbb{R}$ such that $0 \prec \ell_{N} I_{n} \preceq N$. Moreover, the fact that $M \in \mathbb{S}^{n}$ yields that there exists $u_{M} \in \mathbb{R}$ such that $M \preceq u_{M} I_{n}$ and

$$
\ell_{N} M \preceq u_{M} \ell_{N} I_{n} .
$$

Since $u_{M} \in \mathbb{R}$ and $\ell_{N}>0$, there exists $\eta>0$ such that $u_{M}+$ $\eta \ell_{N}>0$. Indeed, it is enough to take $\eta=\frac{\left|u_{M}\right|}{\ell_{N}}+1$. From $\eta>0$ and $\ell_{N}>0$ it follows that

$$
u_{M} \ell_{N}<\left(u_{M}+\eta \ell_{N}\right) \ell_{N} .
$$

Inequalities (13) and (14) yields $\ell_{N} M \prec\left(u_{M}+\eta \ell_{N}\right) \ell_{N} I_{n} \preceq$ $\left(u_{M}+\eta \ell_{N}\right) N$. Since $\ell_{N} \succ 0$, it is enough to take

$$
\mu=\frac{u_{M}+\eta \ell_{N}}{\ell_{N}}=\frac{u_{M}+\left|u_{M}\right|}{\ell_{N}}+1
$$

\section{ACKNOWLEDGEMENTS}

The authors would like to thank the anonymous reviewers for the excellent suggestions and comments which led to significant improvements of the paper. We are grateful to Prof. Jaka Cimprič and Igor De Sant'Ana Fontana for useful discussions. This work received support from the Brazilian agencies CNPq and CAPES.

\section{REFERENCES}

[1] M. C. de Oliveira and R. E. Skelton, "Stability tests for constrained linear systems," in Perspectives in Robust Control (S. O. Reza Moheimani, ed.), vol. 268 of Lecture Notes in Control and Information Science, pp. 241257, New York, NY: Springer-Verlag, 2001.

[2] R. E. Skelton, T. Iwasaki, and K. Grigoriadis, A Unified Algebraic Approach to Linear Control Design. Taylor \& Francis, 1998.

[3] S. Boyd, L. El Ghaoui, E. Feron, and V. Balakrishnan, Linear Matrix Inequalities in System and Control Theory. Philadelphia, PA: SIAM Studies in Applied Mathematics, 1994.

[4] L. El Ghaoui and S. I. Niculescu, eds., Advances in Linear Matrix Inequality Methods in Control. Advances in Design and Control, Philadelphia, PA: SIAM, 2000.

[5] G. R. Duan and H. H. Yu, LMIs in Control Systems: Analysis, Design and Applications. CRC Press, 2013.

[6] P. Gahinet, A. Nemirovskii, A. J. Laub, and M. Chilali, LMI Control Toolbox User's Guide. Natick, MA: The Math Works, 1995.

[7] J. F. Sturm, "Using SeDuMi 1.02, a MATLAB toolbox for optimization over symmetric cones," Optim. Method Softw., vol. 11, no. 1-4, pp. 625653, 1999. http://sedumi.ie.lehigh.edu/
[8] A. Ben-Tal and A. Nemirovski, "Robust convex optimization," Mathematics of Operations Research, vol. 23, no. 4, pp. 769-805, 1998.

[9] P. Apkarian and H. D. Tuan, "Parametrized LMIs in control theory," SIAM J. Control Optim., vol. 38, pp. 1241-1264, May 2000.

[10] P.-A. Bliman, "An existence result for polynomial solutions of parameter-dependent LMIs," Syst. Control Letts., vol. 51, pp. 165-169, Mar. 2004.

[11] R. C. L. F. Oliveira and P. L. D. Peres, "Parameter-dependent LMIs in robust analysis: Characterization of homogeneous polynomially parameterdependent solutions via LMI relaxations," IEEE Trans. Autom. Control, vol. 52, pp. 1334-1340, July 2007.

[12] Y. Ebihara, D. Peaucelle, and D. Arzelier, S-Variable Approach to LMI-Based Robust Control. Communications and Control Engineering, Springer, 2015

[13] Y. Ebihara, T. Hagiwara, D. Peaucelle, and D. Arzelier, "Robust performance analysis of linear time-invariant uncertain systems by taking higher-order time-derivatives of the state," in Proc. 44th IEEE Conf. Decision Control - Eur. Control Conf. ECC 2005, pp. 5030-5035, 2005.

[14] V. Suplin, E. Fridman, and U. Shaked, " $H_{\infty}$ control of linear uncertain time-delay systems-a projection approach," IEEE Trans. Autom. Control, vol. 51, no. 4, pp. 680-685, 2006.

[15] V. F. Montagner, R. C. L. F. Oliveira, and P. L. D. Peres, "Convergent LMI relaxations for quadratic stabilizability and $\mathscr{H}_{\infty}$ control for TakagiSugeno fuzzy systems," IEEE Trans. Fuzzy Syst., vol. 17, pp. 863-873, August 2009.

[16] L. I. Allerhand and U. Shaked, "Robust stability and stabilization of linear switched systems with dwell time," IEEE Trans. Autom. Control, vol. 56, no. 2, pp. 381-386, 2011.

[17] V. Estrada-Manzo, Z. Lendek, and T. M. Guerra, "Output feedback control for TS discrete-time nonlinear descriptor models," in Proc. 53rd IEEE Conf. Decision Control, pp. 860-865, 2014.

[18] L. Frezzatto, M. J. Lacerda, R. C. L. F. Oliveira, and P. L. D. Peres, "Robust $\mathscr{H}_{\infty}$ memory filters for uncertain discrete-time linear systems," in Proc. 2015 Amer. Control Conf., pp. 4004-4009, 2015.

[19] T. M. Guerra, V. Estrada-Manzo, and Z. Lendek, "Observer design for Takagi-Sugeno descriptor models: An LMI approach," Automatica, vol. 52, pp. 154-159, 2015.

[20] J. Cimprič, "Finsler's lemma for matrix polynomials," Lin. Alg. Appl., vol. 465, pp. 239-261, 2015.

[21] P.-A. Bliman, R. C. L. F. Oliveira, V. F. Montagner, and P. L. D. Peres, "Existence of homogeneous polynomial solutions for parameterdependent linear matrix inequalities with parameters in the simplex," in Proc. 45th IEEE Conf. Decision Control, (San Diego, CA, USA), pp. 1486-1491, Dec. 2006.

[22] H. T. M. Kussaba, J. Y. Ishihara, and R. A. Borges, "Uniform versions of Finsler's lemma," in Proc. 54th IEEE Conf. Decision Control, pp. 7292 7297, 2015.

[23] Y. Zi-Zong and G. Jin-Hai, "Some equivalent results with Yakubovich's S-lemma," SIAM J. Control Optim., vol. 48, no. 7, pp. 4474-4480, 2010.

[24] A. A. Albert, "A quadratic form problem in the calculus of variations," Bull. Amer. Math. Soc., vol. 44, no. 4, pp. 250-253, 1938.

[25] W. T. Reid, "A theorem on quadratic forms," Bull. Amer. Math. Soc., vol. 44, no. 6, pp. 437-440, 1938

[26] K. J. Arrow, F. J. Gould, and S. M. Howe, "A general saddle point result for constrained optimization," Math. Program., vol. 5, no. 1, pp. 225234,1973

[27] F. Uhlig, "A recurring theorem about pairs of quadratic forms and extensions: A survey," Lin. Alg. Appl., vol. 25, no. 1, pp. 219-237, 1979.

[28] C. Hamburger, "Two extensions to Finsler's recurring theorem," Appl. Math. Optim., vol. 40, no. 2, pp. 183-190, 1999.

[29] I. Pólik and T. Terlaky, "A survey of the S-lemma," SIAM Rev., vol. 49, no. 3, pp. 371-418, 2007.

[30] P. Finsler, "Über das vorkommen definiter und semidefiniter formen in scharen quadratischer formen," Comment. Math. Helv., vol. 9, pp. 188192, 1937.

[31] G. Pipeleers, B. Demeulenaere, J. Swevers, and L. Vandenberghe, "Extended LMI characterizations for stability and performance of linear systems," Syst. Control Letts., vol. 58, pp. 510-518, July 2009.

[32] R. K. Sundaram, A first course in optimization theory. Cambridge University Press, 1996.

[33] I. R. Manchester, J. Z. Tang, and J.-J. E. Slotine, "Unifying classical and optimization-based methods for robot tracking control with control contraction metrics," in Proc. 2015 Int. Symp. Rob. Res., 2015.

[34] S. Willard, General topology. New York: Dover, 2004.

[35] M. H. Stone, "A generalized Weierstrass approximation theorem," in MAA Studies in Mathematics (R. C. Buck, ed.), pp. 30-87, New Jersey: Prentice-Hall, 1962. 